\title{
CAPILLARY ELECTROPHORETIC ANALYSIS OF EXHALED BREATH CONDENSATE IN THE DIAGNOSIS OF GASTROESOPHAGEAL REFLUX DISEASE
}

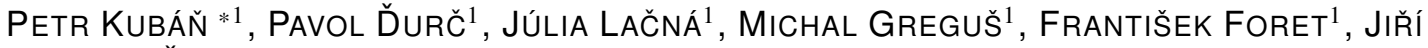

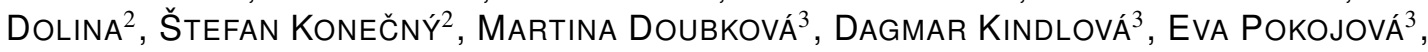 \\ AND JANA SKŘIČKOVÁ ${ }^{3}$ \\ ${ }^{1}$ Department of Bioanalytical Instrumentation, CEITEC Masaryk University, Veveří 97, 60200 Brno, CZECH \\ REPUBLIC \\ ${ }^{2}$ Department of Internal Gastroenterology, Faculty Hospital Brno, Faculty of Medicine, Masaryk University, \\ Jihlavská 340/20, 62500 Brno, CZECH REPUBLIC \\ ${ }^{3}$ Department of Pulmonary Diseases and Tuberculosis, Faculty Hospital Brno, Faculty of Medicine, Masaryk \\ University, Jihlavská 340/20, 62500 Brno, CZECH REPUBLIC
}

\begin{abstract}
In this work, capillary electrophoresis with contactless conductometric detection (CCD) was used for the analysis of the ionic content of exhaled breath condensate $(E B C)$ to differentiate between healthy individuals and patients with gastroesophageal reflux disease (GERD). The exhaled breath condensate was collected using a miniature sample collection device and the content analyzed using a separation electrolyte composed of $20 \mathrm{mM}$ 2-( $\mathrm{N}$-morpholino)ethanesulfonic acid, $20 \mathrm{mM}$ L-histidine, $2 \mathrm{mM}$ 18-Crown- 6 and $30 \mu \mathrm{M}$ cetyltrimethylammonium bromide. The separation of anions took less than 2.5 minutes, while the cations were separated in less than 1.5 minutes. The most significantly elevated ions in the group of patients suffering from gastroesophageal reflux disease were chloride, nitrate, propionate and butyrate. Although the number of subjects was too small to draw definite conclusions with regard to the discriminatory power of these ions, the pilot data are promising for EBC as a useful non-invasive alternative for other methods used in the diagnosis of gastroesophageal reflux disease.
\end{abstract}

Keywords: capillary electrophoresis, exhaled breath condensate, ionic analysis, diagnosis, gastroesophageal reflux disease

\section{Introduction}

Capillary electrophoresis is an attractive separation technique that is particularly suitable for the analysis of biological samples. Non-invasive sampling and the analysis of alternative biofluids are gaining scientific attention as these samples are acquired easily, can be obtained repeatedly without any particular stress to the patient, and often are formed of a simple matrix. One such sample is exhaled breath condensate (EBC).

EBC is obtained by cooling exhaled breath using suitable pieces of cooling equipment and apparatus. EBC is the aqueous part of the exhaled breath that mainly contains condensed water from breath and volatile compounds that are soluble in water, in addition to small respiratory droplets that provide information concerning the condition, inflammation and oxidative/nitrosative stress of the lungs. EBC was first used as a diagnostic sample by Sidorenko in the 1980s [1] and since then numerous articles on EBC have been published, including some re-

\footnotetext{
*Correspondence: petr.kuban@gmail.com
}

cent reviews [2,3]. EBC is attractive not only in terms of studying the condition of the lungs $[4,5]$, but possibly in the identification of other applications, for instance, in the assessment of inflammatory conditions of the trachea and esophagus. The latter is of particular importance because gastroesophageal reflux disease (GERD) is one of the most common diseases in the western world. Between 20 and $30 \%$ of the general population in Europe and USA will suffer from GERD [6] and about $60 \%$ of the adult population will experience some type of GERD during their lifespan. GERD is often diagnosed using invasive instruments such as 24-hour multichannel intraluminal impedance and $\mathrm{pH}$ monitoring (MII-pH) [7], during which the patient is required to continuously wear a narrow catheter in the esophagus. This device measures the spread and volume of gases, liquids as well as solids through the esophagus and evaluates the composition of refluxate. The data are eventually used to evaluate whether the person is suffering from the disease based on the number of recorded reflux episodes and the DeMeester score [8] which describes the severity of reflux. 
The use of MII-pH is, however, rather unpleasant and up to one third of subjects report discomfort. In this paper, the replacement of the above-mentioned MII-pH method with a significantly less invasive technique for analyzing EBC was attempted. EBC was collected using a specially designed miniature sampler $[4,9,10]$ that was previously developed in our laboratory. EBC samples were collected from several healthy volunteers and a group of patients suffering from symptoms attributed to gastroesophageal reflux disease. Capillary electrophoresis was used to analyze the ionic content of EBC samples and differences in selected ion concentrations were identified between the groups.

\section{Experimental}

\subsection{Instrumentation}

An in-house-built CE instrument was used for all electrophoretic separations at $\pm 15 \mathrm{kV}$ using a high voltage power supply unit (Spellman CZE2000R Start Spellman, Pulborough, UK). Two Pt wires (with an outer diameter (OD) of $0.5 \mathrm{~mm}, 3 \mathrm{~cm}$ in length, Advent Research Materials Ltd., Eynsham, UK) were inserted into the electrolyte vials to serve as electrodes. Fused-silica (FS) capillaries (with an inner diameter (ID) of $50 \mu \mathrm{m}$, OD of $375 \mu \mathrm{m}, 39 \mathrm{~cm}$ in total length, Polymicro Technologies, Phoenix, AZ, USA) were used for the separation. All CE experiments were performed at ambient temperature. A custom-made contactless conductometric detector (CCD) (Version 5.06, ADMET s.r.o., Prague, Czech Republic) operating at a frequency of $1.8432 \mathrm{MHz}$ and a voltage of $50 \mathrm{~V}_{\mathrm{p}-\mathrm{p}}$ was used for the detection of the separated analytes. Data were collected using an Orca 2800 24-bit A/D converter and ECOMAC software ver. 0.254 (ECOM spol s.r.o., Prague, Czech Republic). In all experiments, hydrodynamic injection was applied to the samples, consisting of the elevation of the sample vial to a height of $10 \mathrm{~cm}$ for $20 \mathrm{~s}$.

The sampler for the collection of EBC has been developed in our group previously [9]. Briefly, it was constructed from a $2 \mathrm{ml}$ syringe (B. Braun Melsungen AG, Melsungen, Germany) cooled by a $5 \mathrm{~cm}$-long hollow aluminum cylinder with an OD of $2.5 \mathrm{~cm}$ and an ID identical to the OD of the syringe (see Fig. 1).

The cylinder was kept in dry ice at $-80{ }^{\circ} \mathrm{C}$ prior to the collection of $\mathrm{EBC}$ and an insulation sleeve was used during its collection. A straw with an OD of $6 \mathrm{~mm}$, and a wall thickness of $0.2 \mathrm{~mm}$ (purchased in a local store) was used to exhale the air through the sampler. The end of the syringe was enclosed with a parafilm septum to avoid loss of EBC.

\subsection{Chemicals}

All chemicals were of reagent grade (Sigma-Aldrich, Steinheim, Germany) and deionized (DI) water (Purite,
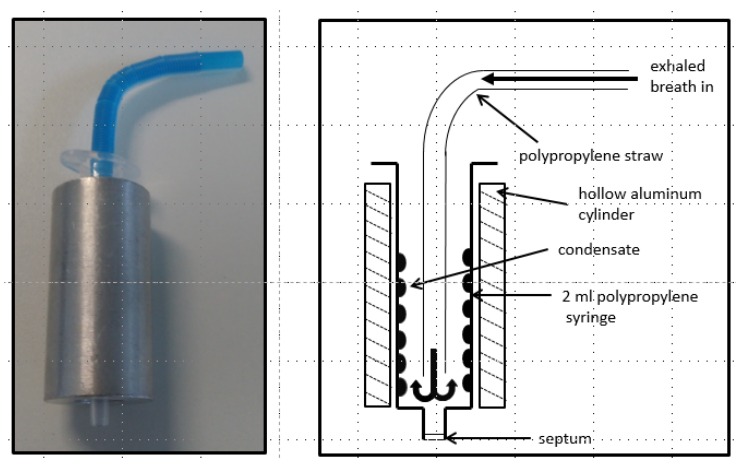

Figure 1: The photograph and scheme of the EBC sampler used in this work.

Neptune, Watrex, Prague, Czech Republic) was used for the preparation of stock solutions and dilutions. Background electrolytes (BGEs) for CE measurements were prepared daily by diluting $100 \mathrm{mM}$ of stock solutions of 2-(N-morpholino)ethanesulfonic acid (MES), L-histidine (L-His) and 18-Crown-6 to the required concentrations. Cetyltrimethylammonium bromide (CTAB) was prepared as a $10 \mathrm{mM}$ stock solution in $5 \%$ acetonitrile.

The separation electrolyte that was used for separation and quantitation of inorganic anions, cations and organic acids consisted of $20 \mathrm{mM}$ MES, $20 \mathrm{mM}$ L-His, 2 $\mathrm{mM}$ 18-Crown-6 and $30 \mu \mathrm{M}$ CTAB.

\subsection{Capillary-conditioning procedure}

Prior to its first use, the separation capillary was preconditioned by flushing it with $0.1 \mathrm{M} \mathrm{NaOH}$ for 30 mins., then with DI water for 30 mins. and finally with a solution of BGEs for 10 mins. Between two successive injections, the capillary was flushed with the BGE solution for $1 \mathrm{~min}$. At the end of a working day, the capillaries were washed with DI water for 15 mins., followed by the application of a vacuum for 5 mins. before being stored dry overnight.

\subsection{Sample-collection procedure}

The EBC was collected in the morning, the subjects did not eat or drink beforehand. The EBC was collected using the previously described EBC-sampler. The subject was asked to exhale deeply 3-5 times into the sampler, but depending on the lung capacity of each, the number of exhalations was modified to collect approximately $100 \mu \mathrm{l}$ of their EBC. After the sampling of EBC was completed, the condensate from the walls of the syringe was forced to the tip of the syringe by the plunger and transferred to the sample vial for CE analysis. Part of the EBC sample was also used in another experiment published elsewhere, in which the $\mathrm{pH}$ was measured using a microelectrode. 


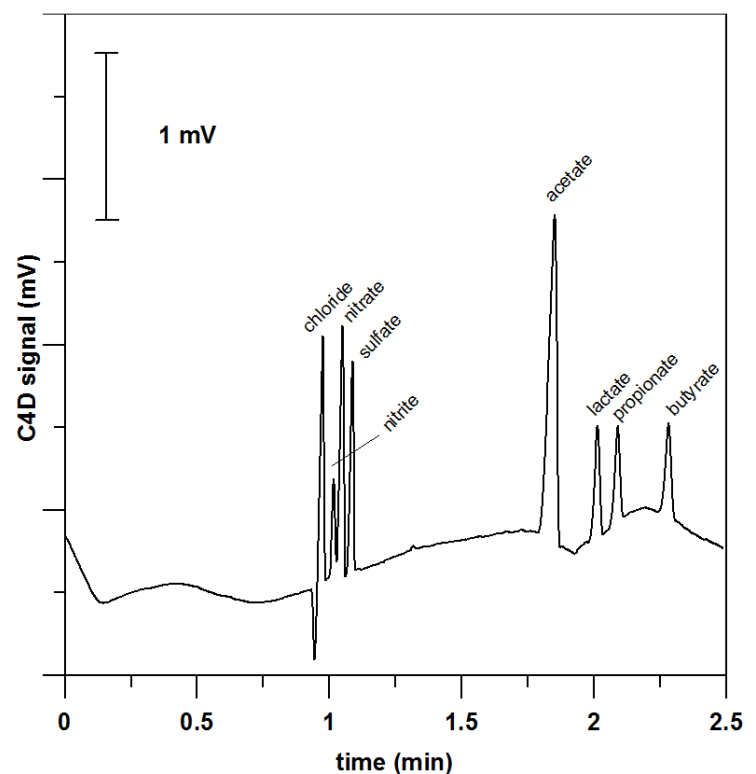

Figure 2: Separation of a model mixture consisting of 4 inorganic anions and 4 organic acids. BGE: $20 \mathrm{mM}$ MES/LHis, 2 mM 18-Crown-6, $30 \mu \mathrm{M}$ CTAB. HV: positive +15 $\mathrm{kV}$, C4D detection. Ion concentrations in the parentheses in $\mu \mathrm{M}$ : acetate (200); chloride, nitrate, lactate, propionate, butyrate (25); nitrite, sulfate (10).

\subsection{Selection of patients and healthy individ- uals}

In this initial screening, a group of healthy individuals and a group of patients diagnosed with asthma and pulmonary fibrosis were selected. The healthy individuals had no history of GERD or heartburn and were free from other related symptoms. The healthy individuals selected were from approximately the same age group as the patients, i.e., the average age of healthy subjects was 48 and that of patients was 56. The patients suffered from asthma and pulmonary fibrosis and were from the Department of Pulmonary Diseases and Tuberculosis at the Faculty Hospital Brno.

\section{Results and Analysis}

\subsection{Electrolyte selection}

To separate the ionic content of the EBC, the BGEs composed of MES and L-His was chosen as this electrolyte allows for the analysis of anions, organic acids, and cations with good degrees of sensitivity. MES and LHis fulfill the criteria for suitable coions and counterions in CE separation using contactless conductivity detection (C4D). The conductance of the respective BGE components is low, thus analytes including small organic acids (acetate, lactate, butyrate) will be detected as positive peaks. The addition of 18 -Crown- 6 to the separation electrolyte improves the resolution of $\mathrm{K}^{+}$and $\mathrm{NH}_{4}^{+}$cations

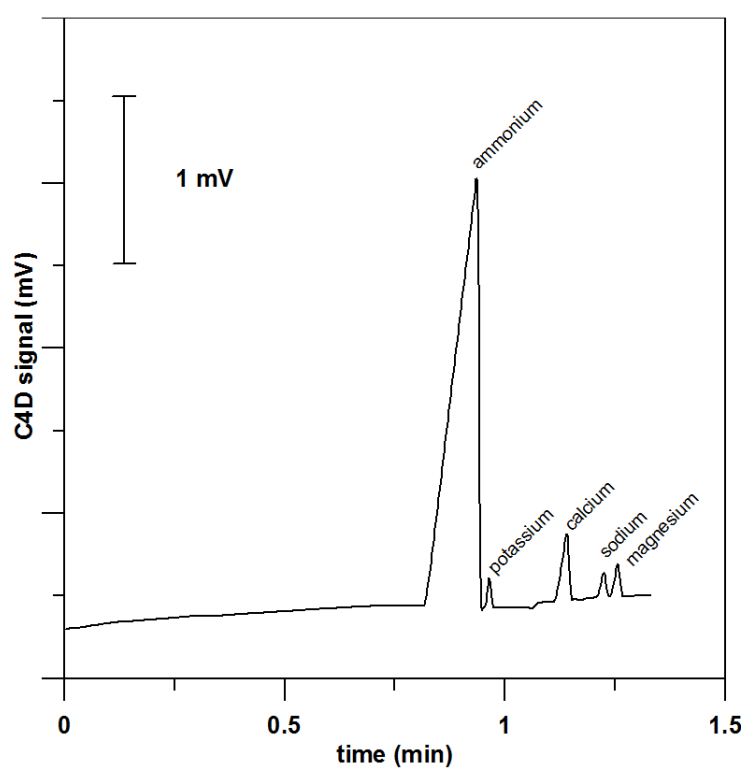

Figure 3: Separation of a model mixture of 5 inorganic cations. BGE: $20 \mathrm{mM}$ MES/L-His, $2 \mathrm{mM}$ 18-Crown-6, 30 $\mu \mathrm{M}$ CTAB. HV: negative $-15 \mathrm{kV}$, C4D detection. Ion concentrations in the parentheses in $\mu \mathrm{M}$ : ammonium (1000); calcium (25); potassium, sodium and magnesium (10).

and does not influence the selectivity of other measured cations to a great extent, nor does it influence the separation of anions and organic acids. CTAB is added to the separation electrolyte to decrease the electroosmotic flow (EOF).

It should be noted here that although this separation electrolyte allows for the simultaneous separation of anions and cations using dual-opposite end injection [11], in this work anions and cations were determined separately by switching the high-voltage polarity. The separation of a model mixture consisting of 8 anions in less than 2.5 mins. is shown in Fig. 2.

The ion concentrations were selected to be similar to those found in EBC samples. The separation of cations using the same conditions but using reverse-polarity voltage is shown in Fig. 3. The ammonium cation is usually present at a concentration 100 times greater than other cations in the EBC, its concentration was thus also increased to $1000 \mu \mathrm{M}$ to reflect the expected concentration. Note that even with this significant excess the separation of $\mathrm{NH}_{4}^{+}$from $\mathrm{K}^{+}$is sufficient and the BGE composition can be used for real sample analysis.

\subsection{Analysis of anions in EBC samples}

From each subject, the EBC sample was collected as described previously. About $50 \mu \mathrm{l}$ of the sample was transferred into an Eppendorf tube and hydrodynamically injected into the CE system. The EBC samples were analyzed first for anions and organic acids using positive $\mathrm{HV}$ polarity and then for cations using negative HV polarity, 


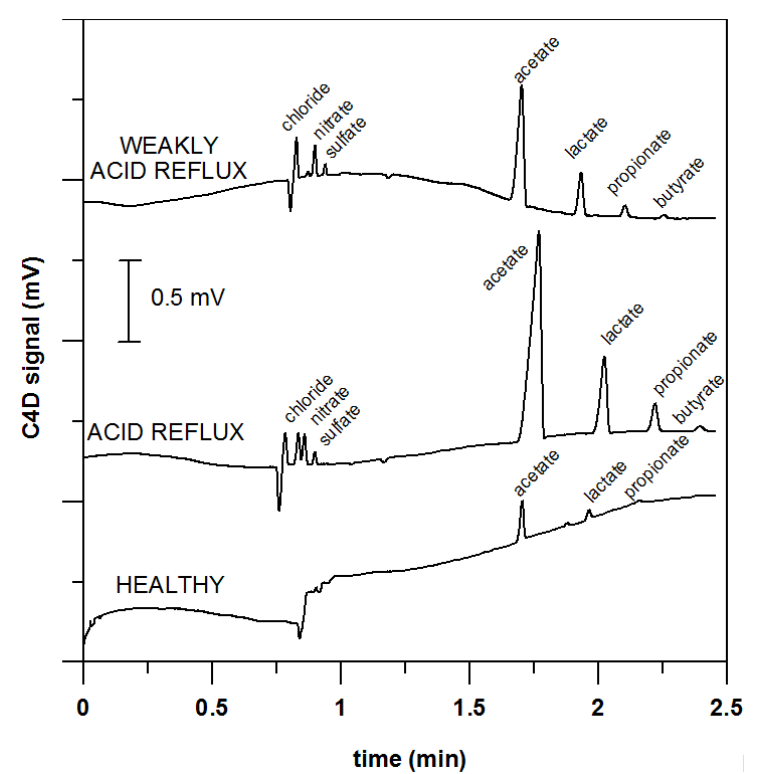

Figure 4: Separation of anions in EBC samples of healthy persons as well as patients with acid reflux or weakly acid reflux. BGE: $20 \mathrm{mM}$ MES/L-His, $2 \mathrm{mM}$ 18-Crown-6, 30 $\mu \mathrm{M}$ CTAB. HV: positive $+15 \mathrm{kV}, \mathrm{C} 4 \mathrm{D}$ detection.

with the HV electrode placed in the detection vial. The concentrations of all 13 ions in the samples were evaluated using calibration curves measured with standard solutions. The parameters such as the calibration curve, regression coefficient $\left(R^{2}\right)$ and concentration range are shown in Table 1. In this initial screening the analysis of several samples from the healthy group and patients that were diagnosed as having the acid reflux or weakly acid reflux was attempted and the ions that would be significantly different in these groups identified.

A series of electropherograms of anions and organic acids in a healthy individual, patient with acid reflux and patient with weakly acid reflux is shown in Fig. 4. One can clearly see that the concentrations of several anions, most notably chloride, nitrate and organic acids, are significantly higher in the group of patients than in that of the healthy volunteers. Some small inorganic anions such as nitrite and nitrate are often found in the EBC of patients with respiratory diseases as markers of nitrosative stress. On the other hand, the presence of an increased concentration of chloride and organic acids may be a significant step towards the diagnosis of GERD.

\subsection{Analysis of cations in EBC samples}

The analysis of cations in the same samples can be seen in Fig. 5. Although higher concentrations of the ammonium cation were found in patients compared to healthy individuals, the content was not significantly different from that of healthy individuals, and this applied for other cations $\left(\mathrm{K}^{+}, \mathrm{Na}^{+}, \mathrm{Ca}^{2+}, \mathrm{Mg}^{2+}\right)$ as well.

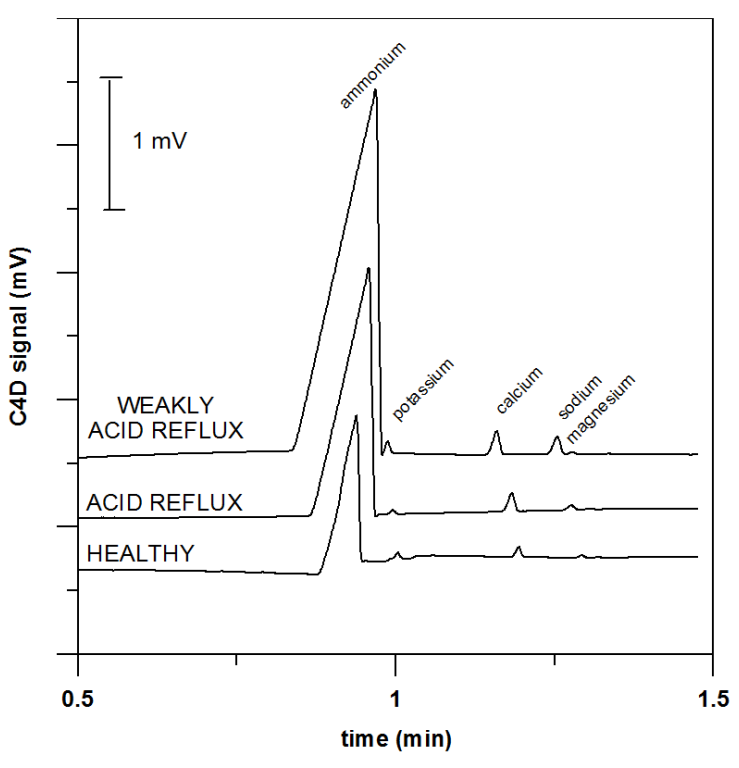

Figure 5: Separation of cations in EBC samples of healthy persons as well as those with acid reflux and weakly acid reflux. BGE: 20 mM MES/L-His, 2 mM 18-Crown-6, 30 $\mu \mathrm{M}$ CTAB. HV: negative $-15 \mathrm{kV}$, C4D detection.

\section{Discussion}

A limited number of subjects were tested and the determination of whether a simple EBC sample could be used as a surrogate for more invasive, expensive and tedious diagnostic methods attempted. The analysis of anions showed some promising results with regard to the small inorganic anions (chloride and nitrate) collected and the concentration of organic acids that were elevated in the patients

Table 1: Calibration equations of all separated anions, cations and organic acids. BGE: $20 \mathrm{mM}$ MES/L-His, 2 mM 18-Crown-6, $30 \mu \mathrm{M}$ CTAB. HV: positive $+15 \mathrm{kV}$ or negative $-15 \mathrm{kV}, \mathrm{C} 4 \mathrm{D}$ detection.

\begin{tabular}{llll}
\hline Ion & Calibration equation & $R^{2}$ & $\begin{array}{l}\text { range } \\
(\mu \mathrm{M})\end{array}$ \\
\hline $\mathrm{Cl}^{-}$ & $y=0.0278 x+0.0258$ & 0.9957 & $0.6-25$ \\
$\mathrm{NO}_{2}^{-}$ & $y=0.0270 x+0.0023$ & 0.9994 & $0.6-25$ \\
$\mathrm{NO}_{3}^{-}$ & $y=0.0315 x+0.0166$ & 0.9987 & $0.6-25$ \\
$\mathrm{SO}_{4}^{2-}$ & $y=0.0656 x+0.0143$ & 0.9980 & $0.25-10$ \\
acetate & $y=0.0156 x+0.0200$ & 0.9995 & $1-200$ \\
lactate & $y=0.0207 x+0.0097$ & 0.9996 & $0.6-25$ \\
propionate & $y=0.0189 x+0.0024$ & 0.9983 & $0.6-25$ \\
butyrate & $y=0.0188 x-0.0001$ & 0.9990 & $0.6-25$ \\
$\mathrm{NH}_{4}^{+}$ & $y=0.0211 x-0.1437$ & 0.9993 & $25-1000$ \\
$\mathrm{~K}^{+}$ & $y=0.0347 x+0.0013$ & 0.9994 & $0.25-10$ \\
$\mathrm{Ca}^{2+}$ & $y=0.0528 x+0.0059$ & 0.9995 & $0.6-25$ \\
$\mathrm{Na}^{+}$ & $y=0.0356 x+0.0079$ & 0.9996 & $0.25-10$ \\
$\mathrm{Mg}^{2+}$ & $y=0.0618 x-0.0054$ & 0.9995 & $0.25-10$ \\
\hline
\end{tabular}


suffering from GERD symptoms, compared to healthy volunteers. Unfortunately, with regard to the analysis of cations, the differences were not significant and cations were deemed unsuitable.

\section{Conclusion}

Capillary electrophoretic analysis of EBC was used for the first time in an attempt to distinguish the groups of patients with GERD from healthy individuals. CE with C4D was used for the analysis of small inorganic anions, cations and organic acids present in the EBC samples. Although it has been shown that selected samples had elevated concentrations of chloride, nitrate, butyrate and propionate, the number of subjects was too small to draw definite conclusions concerning the discriminatory power of these ions. It also seems that although there is a difference between groups of healthy individuals and sufferers of acid reflux/weakly acid reflux, the results with regard to acid reflux and weakly acid reflux are very similar and these two groups cannot be distinguished based on the current data. A larger scale clinical study, in which the EBC of patients and healthy individuals is scrutinized with regard to the ion content, $\mathrm{pH}$ and concentration of other biomarkers (for instance pepsin in saliva) to obtain statistically significant results for evaluation is currently being undertaken. Nevertheless, the initial data is somewhat promising for $\mathrm{EBC}$ as a useful non-invasive alternative for other methods used in GERD diagnostics.

\section{Symbols}

$\begin{array}{ll}\text { BGE } & \text { background electrolyte } \\ \text { CE } & \begin{array}{l}\text { capillary electrophoresis } \\ \text { contactless conductivity detection }\end{array} \\ \text { C4D } & \begin{array}{l}\text { cetyltrimethylammonium bromide } \\ \text { CTAB }\end{array} \\ \text { DI } & \begin{array}{l}\text { deionized } \\ \text { EBh }\end{array} \\ \text { GERD } & \text { gastroesophageal reflux disease } \\ \text { L-His } & \text { L-histidine } \\ \text { ID } & \text { inner diameter } \\ \text { MES } & \text { 2-(N-morpholino)ethanesulfonic acid } \\ \text { OD } & \text { outer diameter }\end{array}$

\section{Acknowledgement}

This research was supported by grant no. 17-31945A from the Ministry of Health of the Czech Republic.

\section{REFERENCES}

[1] Sidorenko, G.I.; Zborovskii, E.I.; Levina, D.I.: Surface-active properties of the exhaled air condensates (a new method of studying lung function), Ter. Arkh., 1980 52, 65-68
[2] Kubáň, P.; Foret, F.: Exhaled breath condensate: Determination of non-volatile compounds and their potential for clinical diagnosis and monitoring. A review, Anal. Chim. Acta, 2013 805, 1-18 DOI: 10.1016/j.aca.2013.07.049

[3] Hayes, S.A.; Haefliger, S.; Harris, B.; Pavlakis, N.; Clarke, S.J.; Molloy, M.P.; Howell, W.M.: Exhaled breath condensate for lung cancer protein analysis: a review of methods and biomarkers, J. Breath Res., 2016 10(3), 034001 DOI: 10.1088/1752-7155/10/3/034001

[4] Greguš, M.; Foret, F.; Kindlová, D.; Pokojová, E.; Plutinsk $\imath$, M.; Doubková, M.; Merta, Z.; Binková, I.; Skřičková, J.; Kubáň, P.: Monitoring the ionic content of exhaled breath condensate in various respiratory diseases by capillary electrophoresis with contactless conductivity detection, J. Breath Res., 2015 9, 027107 DOI: $10.1088 / 1752-7155 / 9 / 2 / 027107$

[5] Aldakheel, F.M.; Thomas, P.S.; Bourke, J.E.; Matheson, M.C.; Dharmage, S.C.; Lowe, A.J.: Relationships between adult asthma and oxidative stress markers and $\mathrm{pH}$ in exhaled breath condensate: a systematic review, Allergy, 2016 71(6), 741-757 DOI: 10.1111/all.12865

[6] El-Serag, H.B.; Sweet, S.; Winchester, C.C.; Dent, J.: Update on the epidemiology of gastro-oesophageal reflux disease: a systematic review, Gut., 2014 63(6), 871-880 DOI: 10.1136/gutjnl-2012-304269

[7] Tutuian, R.; Castell, D.O.: Review Article: complete gastro-oesophageal reflux monitoring- combined $\mathrm{pH}$ and impedance, Aliment. Pharmacol. Ther., 2006 2, 27-37 DOI: 10.1111/j.1365-2036.2006.03039.x

[8] Johnson, L.F.; DeMeester, T.R.: Twenty-four-hour $\mathrm{pH}$ monitoring of the distal esophagus. A quantitative measure of gastroesophageal reflux, Am. J. Gastroenterol., 1974 62, 325-332

[9] Greguš, M.; Foret, F.; Kubáň, P.: Single-breath analysis using a novel simple sampler and capillary electrophoresis with contactless conductometric detection, Electrophoresis, 2015 36(4), 526-533 DOI: 10.1002/elps.201400456

[10] Greguš, M.; Foret, F.; Kubáň, P.: Portable capillary electrophoresis instrument with contactless conductivity detection for on-site analysis of small volumes of biological fluids, J. Chromatogr. A, 2016 14(27), 177-185 DOI: 10.1016/j.chroma.2015.11.088

[11] Kuban, P.; Karlberg, B.; Kuban, P.; Kuban, V.: Application of a contactless conductometric detector for the simultaneous determination of small anions and cations by capillary electrophoresis with dualopposite end injection, J. Chromatogr. A, 2002 964, 227-241 DOI: 10.1016/S0021-9673(02)00656-8 